SWAT/396

SMUHEP/04-06

Revised May 2005

\title{
Spontaneously broken quark helicity symmetry
}

\author{
Simon Dalley \\ Department of Physics, University of Wales Swansea \\ Singleton Park, Swansea SA2 8PP, United Kingdom \\ Gary McCartor \\ Department of Physics, SMU \\ Dallas, TX 75275
}

\begin{abstract}
We discuss the origin of chiral symmetry breaking in the light-cone representation of QCD. In particular, we show how quark helicity symmetry is spontaneously broken in $S U(N)$ gauge theory with massless quarks if that theory has a condensate of fermion lightcone zero modes. The symmetry breaking appears as induced interactions in an effective lightcone Hamiltonian equation based on a trivial vacuum. The induced interaction is crucial for generating a splitting between pseudoscalar and vector meson masses, which we illustrate with spectrum calculations in some 1+1-dimensional reduced models of gauge theory.
\end{abstract}




\section{INTRODUCTION}

The lightcone Hamiltonian approach to quantum field theories [1] is an intuitively appealing and powerful tool in the study of gauge theories. There has been steady progress in recent years to formulate and solve such theories at the non-perturbative level. In pursuit of this goal, however, one runs up against an apparent paradox. One of the appealing features of lightcone quantization is the simplified vacuum structure - often it is chosen to be the trivial bare vacuum. But in many cases of physical interest, there is supposed to be a complicated vacuum structure. The success of the QCD sum rules [2], for example, suggests that chiral and other condensates play an important role in extracting hadronic physics from QCD. In this paper, we investigate how chiral condensates could enter in the lightcone formulation of QCD and, assuming that they are there, show how this leads to the breaking of quark helicity symmetry with massless quarks. We derive the explicit form of the quark helicity violating interactions, induced into a lightcone QCD hamiltonian acting effectively in a trivial vacuum. It is shown to be necessary for splitting of pseudoscalar from vector meson masses and illustrative non-perturbative calculations are performed in dimensionally reduced models.

The general mechanism by which a trivial light-cone vacuum can become dressed with fermions is now well understood [3, 4, 5, 5] , although detailed representations have been given only in a few simple models. In the cases that are completely understood, all of the physics of the full theory, including the dressed vacuum, can be represented by induced interactions that act in the usual light-cone representation space based on a trivial vacuum. In that sense, the problem is very similar to the standard renormalization problem: degrees of freedom (those that dress the vacuum) are removed from the theory; when those degrees of freedom are properly "integrated out" rather than just ignored, new effects appear that provide the same physics using only the remaining degrees of freedom. Of course, the usual high energy divergences of QCD also appear in the light-cone representation and must be controlled by some sort of regulators. Counterterms associated with those regulation procedures may also add new interactions to the theory. Given the interactions, a number of approaches to renormalization of their couplings have been investigated, such as perturbative similarity renormalization group [7] and non-perturbative symmetry optimization [8].

The fundamental requirement of Lorentz invariance implies that, in the continuum, the 
vacuum state should be the same in any quantization scheme. Therefore, the vacuum in the light-cone representation is the same vector as in the 'equal-time' representation, but the different quantization procedures give that vector in different representations. Likewise, condensates and any other quantity that is representation independent, must be the same whichever quantization scheme is used. When the theory is quantized directly on null-planes, the retention of lightcone zero modes can dress the trivial vacuum and will do so if the theory incorporates a structured vacuum that supports condensates. This is the approach we follow here. Let us note, however, that there exist some other approaches to the lightcone vacuum problem which we do not pursue here, such as the use of near lightcone quantization [9] or point-splitting in lightcone time [10]. The spontaneous violation of helicity has also been investigated in trivial bare lightcone vacua perturbed by mass breaking terms [1]]. For any method, computing the details of the representation of the vacuum in light-cone quantization of QCD would be quite involved. In this paper we have a more modest goal. Imposing the required symmetries on the form of a general chiral-symmetry-breaking vacuum, we derive new effective interactions, operating in a trivial vacuum, that violate quark helicity. The conservation of quark helicity differs from that of chiral charge only by the neglect of zero mode vacuum structure and hence is sensitive to the spontaneous (and anomalous) breaking of chiral symmetry. The violation of quark helicity is crucial for obtaining the correct hadron spectrum, previous literature behind this idea having been reviewed by Mustaki [12].

The organization of this paper is as follows: In the next section we introduce the tools we will need, emphasizing the role of helicity symmetry and rewriting the theory in a way which exposes the zero mode operators that can dress the vacuum. In section of induced interactions that violate quark helicity in massless $S U(N)$ gauge theory, assuming the existence of a chiral symmetry-breaking fermion condensate. To illustrate the effect on spectrum calculations, in particular pseudoscalar-vector splitting, we then perform some computations on 1+1-dimensionally reduced models. We obtain exact solutions to a model containing only the new induced interactions in section IV. Adding gauge interactions, we then perform numerical DLCQ calculations of the meson spectrum. Conclusions are discussed in section $\mathrm{V}$ In Appendix A we briefly review the Schwinger Model, which is an example where the fermionic vacuum structure is exactly known [4]. 


\section{HELICITY AND THE VACUUM}

\section{A. Quark helicity symmetry}

Unless otherwise stated, our metric is such that lightcone coordinates are $x^{ \pm}=x^{0} \pm x^{3}$, $x_{\perp}=\left(x^{1}, x^{2}\right)$, where $x^{+}$is the light-front time variable. We decompose fermion spinors $\psi=$ $\psi_{+}+\psi_{-}$into the left and right moving projections $\psi_{ \pm}=\frac{1}{2} \gamma_{\mp} \gamma_{ \pm} \psi$. Each of these projections can be further decomposed into different helicities $\psi_{ \pm, \uparrow}=\frac{1}{2}\left(1+\gamma_{5}\right) \psi_{ \pm}, \psi_{ \pm, \downarrow}=\frac{1}{2}\left(1-\gamma_{5}\right) \psi_{ \pm}$. Similarly, we define the helicity eigenstates of the transversely polarized gauge fields via $A_{\uparrow}=\left(A_{1}-\mathrm{i} A_{2}\right) / \sqrt{2}, A_{\downarrow}=\left(A_{1}+\mathrm{i} A_{2}\right) / \sqrt{2}$.

In QCD with massless quarks, the action is

$$
S=\int d^{4} x\left[-\frac{1}{4} \operatorname{Tr} F_{\mu \nu} F^{\mu \nu}+\mathrm{i} \bar{\psi} \gamma^{\mu} D_{\mu} \psi\right],
$$

$\mu, \nu \in\{0,1,2,3\}$

$$
D_{\mu}=\partial_{\mu}+\mathrm{i} g A_{\mu}
$$

This action is invariant under the chiral transformation

$$
\psi \rightarrow \exp \left(i \gamma_{5} \theta\right) \psi
$$

We work in light-cone gauge $A_{-}=0$ throughout, in which case $\psi_{-}$satisfies a constraint equation

$$
\mathrm{i} \partial_{-} \psi_{-}=\mathrm{i} \gamma_{\perp} \cdot D_{\perp} \gamma^{0} \psi_{+}
$$

Integrating, we obtain

$$
\psi_{-}=\psi_{-}^{0}\left(x^{+}, x^{\perp}\right)+\int d x^{-} \gamma_{\perp} \cdot D_{\perp} \gamma^{0} \psi_{+}
$$

Here, $\int$ is the antiderivative which just replaces $\mathrm{e}^{\mathrm{i} k x}$ with $\frac{1}{\mathrm{i} k} \mathrm{e}^{\mathrm{i} k x}$ in the Fourier expansion of the field. We see that $\psi_{-}$is not quite a dependent field of $\psi_{+}$. The constant of integration is the $x^{-}$-zero mode $\psi_{-}^{0}\left(x^{+}, x^{\perp}\right)$, which is a field that remains independent of $\psi_{+}$. This zero mode can dress the lightcone vacuum state, breaking chiral symmetry, as we shall see.

In order to cope with fields that may not diminish sufficiently at large distances to discard, when necessary one can place the system in a box of length $2 L$ in both $x^{-}$and $x^{+}$[13]. If a charge is defined as the integral over $x^{0}=0$ of the zero component of a conserved current, one can calculate the same quantity by integrating over the surface illustrated in Figure 1. 


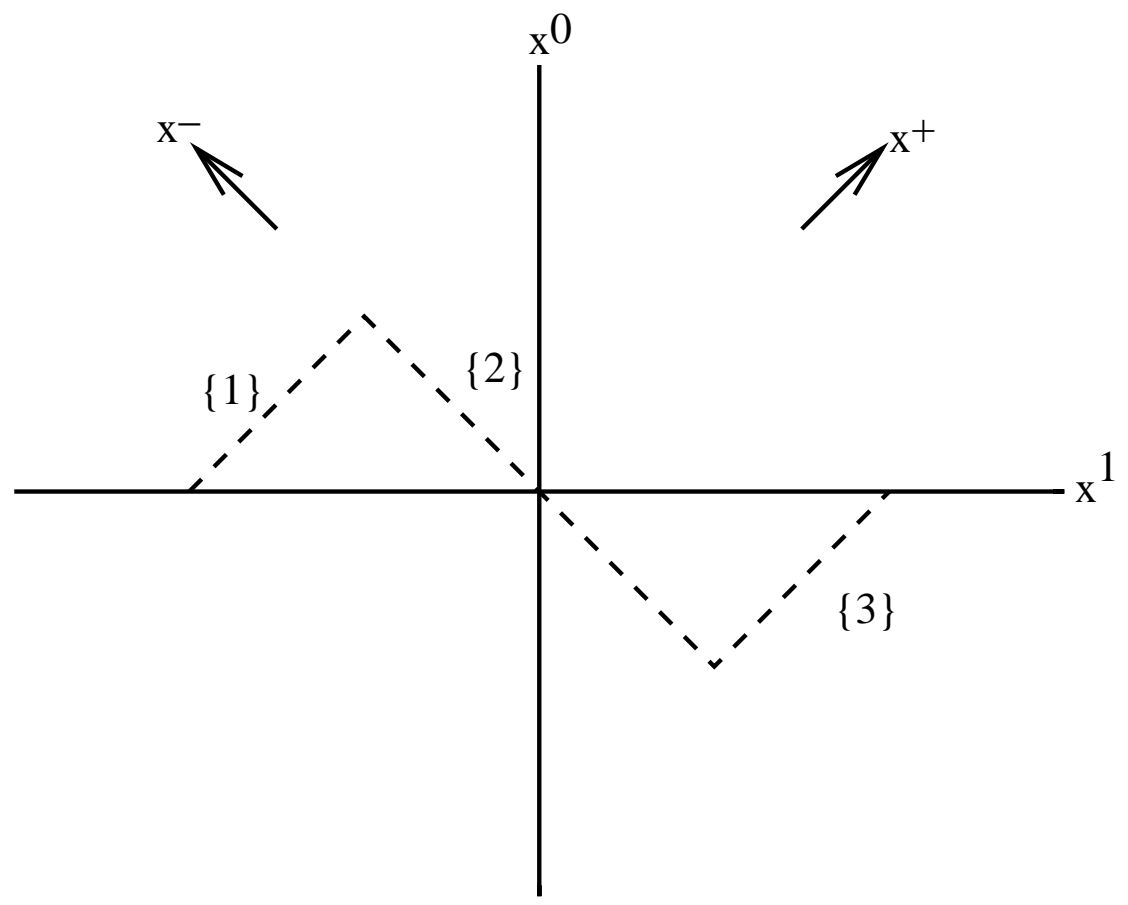

FIG. 1: A null surface for calculating charges. Its extension to $\{1\}$ and $\{3\}$ is necessary to correctly incorporate fields that are independent of $x^{-}$. [13].

This includes not only the portion, labelled $\{2\}$ in figure 1, on which the fields are initialized in the light-cone representation, but also the legs $\{1\}$ and $\{3\}$. In general the integrals over $\{1\}$ and $\{3\}$ do not go to zero as $L$ goes to infinity. Integration constants such as $\psi_{-}^{0}$ cannot be initialized on surface $\{2\}$. Thus, for example, on the $x^{+}=0$ initialization surface $\{2\}$ we have

$$
\psi_{+, s}^{(a)}\left(0, x^{-}, x_{\perp}\right)=\frac{1}{\sqrt{\Omega_{q}}} \int_{0}^{\infty} d k^{+} b_{s}^{(a)}\left(k^{+}, x_{\perp}\right) \mathrm{e}^{-i k^{+} x^{-}}+d_{-s}^{(a) *}\left(k^{+}, x_{\perp},\right) \mathrm{e}^{i k^{+} x^{-}} .
$$

Here $(a)$ is the color index, $s$ the helicity index (such that if $s=\uparrow,-s=\downarrow$ ), and $\Omega_{q}$ a normalization factor chosen so that the anti-commutation relations are

$$
\left\{b_{s_{1}}^{(a)}\left(k^{+}, x_{\perp}\right), b_{s_{2}}^{(b) *}\left(p^{+}, y_{\perp}\right)\right\}=\delta\left(k^{+}-p^{+}\right) \delta\left(x_{\perp}-y_{\perp}\right) \delta_{s_{1} s_{2}} \delta_{a b}
$$

with similar relations for anti-fermions $d$. The independent fermion $\psi_{-}^{0}$ is a function only of $x^{+}$and may be expanded as

$$
\psi_{-, s}^{(0)(a)}\left(x^{+}, x_{\perp}\right)=\frac{1}{\sqrt{\Omega_{q}}} \int_{0}^{\infty} d k^{-} \beta_{s}^{(a)}\left(k^{-}, x_{\perp}\right) \mathrm{e}^{-i k^{-} x^{+}}+\delta_{-s}^{(a) *}\left(k^{-}, x_{\perp},\right) \mathrm{e}^{i k^{-} x^{+}} .
$$

For gluons on $\{2\}$ we write

$$
A_{s}^{(c)}\left(0, x^{-}, x_{\perp}\right)=\frac{1}{\sqrt{\Omega_{g}}} \int_{0}^{\infty} d k^{+} \frac{1}{\sqrt{2 k^{+}}}\left(a_{s}^{(c)}\left(k^{+}, x_{\perp}\right) \mathrm{e}^{-i k^{+} x^{-}}+a_{-s}^{(c)^{*}}\left(k^{+}, x_{\perp},\right) \mathrm{e}^{i k^{+} x^{-}}\right)
$$




$$
\left[a_{s_{1}}^{(b)}\left(k^{+}, x_{\perp}\right), a_{s_{2}}^{(c) *}\left(p^{+}, y_{\perp}\right)\right]=\delta\left(k^{+}-p^{+}\right) \delta\left(x_{\perp}-y_{\perp}\right) \delta_{s_{1} s_{2}} \delta_{a b}
$$

Of course, for finite $L$ the above integrals over $k^{ \pm}$become discrete sums for integers $n$ of $\pi n / L$ (bosons) or $\pi(2 n-1) / 2 L$ (fermions).

Of particular interest in the present case is the chiral charge generator, defined by integrating the zero component of the chiral current

$$
j_{5}^{\mu}=\bar{\psi} \gamma^{\mu} \gamma_{5} \psi
$$

over $x^{0}=0$. If the underlying chiral current is conserved, $\partial_{\mu} j_{5}^{\mu}=0$, then it is also equal to the integral of the normal component of the chiral current over $\{1\},\{2\}$ and $\{3\}$. If we neglect the contribution from surfaces at $x^{-}= \pm L$, one finds

$$
\begin{aligned}
& \int \bar{\psi} \gamma^{+} \gamma_{5} \psi d^{2} x_{\perp} d x^{-} \\
& =\int d x_{\perp} d k^{+} \sum_{s} s\left[b_{s}^{(a) *}\left(k^{+}, x_{\perp}\right) b_{s}^{(a)}\left(k^{+}, x_{\perp}\right)+d_{s}^{(a) *}\left(k^{+}, x_{\perp}\right) d_{s}^{(a)}\left(k^{+}, x_{\perp}\right)\right]
\end{aligned}
$$

which measures the total quark and anti-quark helicity. Note that this is sometimes also called the light-front chiral charge 12]. This light-front chiral charge differs from the chiral charge by the integral of the chiral density over $\{1\}$ and $\{3\} ; \psi_{-}^{0}$ will contribute to these last integrals. If the underlying chiral current is conserved, and there are no non-trivial (vacuum) effects from zero modes on the neglected surfaces, one expects quark helicity to be conserved. However, the non-conservation of quark helicity is crucial to producing the experimentally observed splitting in the meson spectrum between pseudoscalar and vector mesons [7, 14, 15]. To see this in more detail, one notes that the valence quark content of the pseudoscalar and helicity zero component of the vector is $|\uparrow \downarrow\rangle \mp|\downarrow \uparrow\rangle$ respectively. They differ only by being symmetric and anti-symmetric under quark helicity flip. Interactions that conserve quark helicity cannot split them in energy. If the zero mode $\psi_{-}^{0}$ is neglected, the QCD action (11) contains only quark helicity preserving interactions and cannot produce the required splitting. Therefore, we deduce that, in QCD, one cannot neglect the flow of chiral charge across the surfaces at $x^{-}= \pm L$ and one must retain the zero modes that live there. The violation of quark helicity is a direct consequence of conserved chiral current and non-trivial structure of the vacuum which violates spontaneously the chiral symmetry (3). 


\section{B. Dressing the light-cone vacuum}

In order to investigate in more detail the considerations of the previous subsection, we now rewrite the fields in such a way as to expose the operators that can dress the vacuum. In intermediate steps in our analysis we will work with discretized $x_{\perp}$. The fermion at given $x_{\perp}, a$, and $s$ is then just a one dimensional fermi field which can be bosonized in the form 16, 17]

$$
\psi_{+, s}^{(a)}\left(0, x^{-}, x^{\perp}\right)=Z_{+} \mathrm{e}^{-\lambda_{s}^{(a)(-)}\left(x^{-}, x_{\perp}\right)} \sigma_{+, s}^{(a)}\left(x_{\perp}\right) \mathrm{e}^{-\lambda_{s}^{(a)(+)}\left(x^{-}, x_{\perp}\right)},
$$

where $Z_{+}$is a wavefunction renormalization constant,

$$
\begin{gathered}
\lambda_{s}^{(a)(+)}\left(x^{-}, x_{\perp}\right)=-\int_{0}^{\infty} d k^{+} \frac{1}{k^{+}} C_{+, s}^{(a)}\left(k^{+}, x_{\perp}\right)\left(\mathrm{e}^{-i k^{+} x^{-}}-\theta\left(k-k^{+}\right)\right), \\
\lambda_{s}^{(a)(-)}\left(x^{-}, x_{\perp}\right)=-\lambda_{s}^{(a)(+)^{*}} \\
C_{+, s}^{(a)}\left(k^{+}, x_{\perp}\right)=\int_{0}^{k^{+}} d q^{+} d_{-s}^{(a)}\left(q^{+}, x_{\perp}\right) b_{s}^{(a)}\left(k^{+}-q^{+}, x_{\perp}\right)+ \\
\int_{0}^{\infty} d q^{+} b_{s}^{(a) *}\left(q^{+}, x_{\perp}\right) b_{s}^{(a)}\left(k^{+}+q^{+}, x_{\perp}\right)- \\
\int_{0}^{\infty} d q^{+} d_{-s}^{(a) *}\left(q^{+}, k_{\perp}\right) d_{-s}^{(a)}\left(k^{+}+q^{+}, x_{\perp}\right) .
\end{gathered}
$$

and $k$ is a Klaiber regulator [18]. Since the 'fusion' fields $C_{+}$are bosons, $\sigma_{+}$is an $x^{ \pm}$ independent 'spurion' inserted to carry the fermionic quantum numbers of $\psi_{+}$. It is important to appreciate that, in re-writing the fermi field in this way, we have made no assumptions or approximations; the field written in terms of the $C^{\prime}$ 's and $\sigma$ 's is identical to (6). While we will not need most of the details of the fusion fields, the spurions will play a key role. We define a similar bosonization for the $\psi_{-}^{0}$ field in terms of $\sigma_{-}\left(x_{\perp}\right)$ and $C_{-}\left(k^{-}, x_{\perp}\right)$. Since the fusion operator $C_{-}$in this case is independent of $x^{-}\left(k^{+}=0\right)$, it will create free bosons of mass squared $-k_{\perp}^{2}$. These are tachyonic and therefore cannot appear in the physical states of the theory, although they may in general be needed to recover the full canonical structure. The operators we will retain from the $\psi_{-}^{0}$ fields are their 'spurions' $\sigma_{-}$. For convenience, we note here the commutation relations of the spurions that follow from those of the fermi field [1],

$$
\begin{array}{r}
\sigma_{\tau}^{*} \sigma_{\tau}=\sigma_{\tau} \sigma_{\tau}^{*}=1 \\
\left\{\sigma_{\tau}^{*}, \sigma_{\rho}\right\}=\left\{\sigma_{\tau}, \sigma_{\rho}\right\}=0
\end{array}
$$


where $\tau$ and $\rho$ are differing labels indicating a Lorentz, colour, or transverse structure. Spurions commute with the fusion operators.

At this point we can see that the presence of $\psi_{-}^{0}$, in particular the spurion $\sigma_{-}$, allows for dressing of a trivial lightcone bare vacuum $|0\rangle$. Since the fusion operators $C_{+}\left(k^{+}\right)$carry positive definite lightcone momentum $k^{+}$, they cannot appear in the vacuum which should have momentum zero (this is just the usual argument that leads to the trivial light-cone vacuum). The spurions are kinematically allowed to dress the vacuum but considerations of symmetry restrict the possible ways that can occur. The fact that the vacuum must be a chargeless Lorentz scalar implies that, in the sector where there are no gauge-field zero modes, only bilinear combinations of the form $\sigma_{-, s}^{*} \sigma_{+,-s}$, or its conjugate, can occur. Note that these combinations, which are the spurion part of $\bar{\psi} \psi$, are not chirally invariant. Other chargeless Lorentz invariant combinations of spurions can always be reduced to combinations of the previous bilinear forms using the rules (18).

The pure fermion content of a general vacuum state must therefore be of the form

$$
\left|\Omega_{f}\right\rangle=F\left[\sigma_{-, s}^{(a) *}\left(x_{\perp}\right) \sigma_{+,-s}^{(a)}\left(x_{\perp}\right), \sigma_{+,-s}^{(a) *}\left(x_{\perp}\right) \sigma_{-, s}^{(a)}\left(x_{\perp}\right)\right]|0\rangle
$$

If the theory is to be charge conjugation invariant, the functional $F$ must be symmetric in its arguments. The spurion components of the bilinears must also occur with the same colour and at the same transverse position if the vacuum is to be invariant under $x_{\perp}$-dependent gauge transformations (we show later that $\sigma^{(a)}$ transforms in the fundamental representation when it acts on $|0\rangle)$. Lastly, the functional $F$ should be invariant under shifts of $x_{\perp}$ for translation invariance.

If $\left|\Omega_{f}\right\rangle$ is not equal to $|0\rangle$, which we assume in this paper, then it follows that there is a $\bar{\psi} \psi$ condensate from this sector of the theory

$$
\left\langle\bar{\psi} \psi\left(x_{\perp}\right)\right\rangle \sim\left\langle\Omega_{f}\left|\sum_{s, a} \sigma_{-, s}^{(a) *}\left(x_{\perp}\right) \sigma_{+,-s}^{(a)}\left(x_{\perp}\right)+c . c .\right| \Omega_{f}\right\rangle
$$

Translation invariance of the condensate implies that the combination $\sigma_{-, s}^{(a) *}\left(x_{\perp}\right) \sigma_{+,-s}^{(a)}\left(x_{\perp}\right)$ is independent of $x_{\perp}$ in the vacuum. The contribution from $\left|\Omega_{f}\right\rangle$ may not be the total condensate, for sectors of the vacuum containing gluon zero modes could also contribute, i.e. the total vacuum is $|\Omega\rangle=\left|\Omega_{f}\right\rangle+\left|\Omega_{g}\right\rangle$, where every vector in $\left|\Omega_{g}\right\rangle$ contains gluon fields. In fact, for the aim of this paper in demonstrating quark helicity violation, we will not need to consider the sector of the vacuum that includes gluon zero modes or the induced 
interactions that might result from that structure. That is not to say these sectors are physically unimportant, but gluon condensates and the effective interactions they generate is a separate question to the one we address here. Not only do we not need the sector of the vacuum containing gluon zero modes, but to find the form of the induced interaction that violates quark helicity we shall not need to specify the exact form of $F$. There are examples of much simpler field theories where this vacuum functional can be obtained exactly. The most completely solved example is the Schwinger Model, for which we include a brief review in appendix A. For the case of the massive Schwinger model, there is an induced interaction whose form is exactly known [19]. That example serves to demonstrate that the form (19) can lead to induced interactions and, moreover, is sufficiently general to include the case of anomalous chiral symmetry breaking and $\theta$-vacua.

\section{INDUCED INTERACTIONS IN QCD}

The lightcone QCD Hamiltonian $P^{-}$derived from the action (11) satisfies an eigenvalue equation which is the relativistic Schrodinger equation for this problem:

$$
\left(P^{+} P^{-}-P_{\perp}^{2}\right)|\Psi\rangle=M^{2}|\Psi\rangle
$$

$M$ is the invariant mass eigenvalue. The eigenstates, $|\Psi\rangle$, can represent the vacuum, boundstates of quarks and gluons (hadrons), or scattering states of hadrons. Since we focus on the new effects introduced by fermion lightcone zero modes, we do not need all the details

of $P^{-}$. The interested reader is referred to a review such as ref. 1] for a fuller description of the lightcone QCD hamiltonian.

Any interaction in $P^{-}$that depends on $\psi_{-}$can potentially give rise to an induced interaction through the existence of $\psi_{-}^{0}$. For the massless QCD case we consider in this paper, in lightcone gauge the only one is $-\bar{\psi} \gamma_{\perp} i D_{\perp} \psi$. The part of this interaction that depends on $\psi_{-}^{0}$ induces extra, quark-helicity-violating, operators in the QCD action (11) and hence the lightcone hamiltonian. It is given by

$$
\begin{aligned}
I & =I_{1}+I_{2} \\
I_{1} & =\int d x^{-} d^{2} x^{\perp} \sum_{a}\left(i \partial_{\uparrow} \psi_{+, \downarrow}^{(a) *}\right) \psi_{-, \downarrow}^{0(a)}+\text { c.c. }-[\downarrow \leftrightarrow \uparrow] \\
I_{2} & =g \int d x^{-} d^{2} x^{\perp} \sum_{a b c} \lambda_{a b}^{c} \psi_{+, \downarrow}^{(a) *} \psi_{-, \downarrow}^{0(b)} A_{\uparrow}^{(c)}+\text { c.c. }-[\downarrow \leftrightarrow \uparrow]
\end{aligned}
$$


where $\lambda_{a b}^{c}$ is the colour factor ( $a b$ matrix element of the $c^{\text {th }}$ generator of $S U(N)$ ),

$$
\partial_{\uparrow}=\left(\partial_{1}-i \partial_{2}\right) / \sqrt{2}, \partial_{\downarrow}=\partial_{\uparrow}^{*}
$$

and c.c. means complex conjugate. We are going to study the operators that result from the part containing only the spurion from the $\psi_{-}$field. With that restriction, the first term of $I_{2}$, for example, is given by

$$
g Z_{-} \int d x^{-} d^{2} x^{\perp} \sum_{a b c} \lambda_{a b}^{c} \psi_{+, \downarrow}^{(a) *} \sigma_{-, \downarrow}^{(b)} A_{\uparrow}^{(c)}
$$

where $Z_{-}$is an (unknown) renormalization constant that will depend upon the regulator eventually used. From the identities (18), we can insert 1 in the form $\sigma_{+, \uparrow}^{(b) *}\left(x_{\perp}\right) \sigma_{+, \uparrow}^{(b)}\left(x_{\perp}\right)$, to rewrite it as

$$
g Z_{-} \int d x^{-} d^{2} x^{\perp} \sum_{a b c} \lambda_{a b}^{c} A_{\uparrow}^{(c)} \psi_{+, \downarrow}^{(a) *} \sigma_{-, \downarrow}^{(b)} \sigma_{+, \uparrow}^{(b) *} \sigma_{+, \uparrow}^{(b)} .
$$

We now commute the spurions among themselves to get a combination on the far right, shown in square brackets below, that is in the same form as those appearing in the purefermion part of the vacuum state (19). Proceeding in this way also for all the parts of $I_{2}$, we obtain:

$$
\begin{aligned}
I_{2}= & g Z_{-} \int d x^{-} d^{2} x^{\perp} \sum_{a b c} \lambda_{a b}^{c}\left\{A_{\uparrow}^{(c)} \psi_{+, \downarrow}^{(a) *} \sigma_{+, \uparrow}^{(b)}\left[\sigma_{+, \uparrow}^{(b) *} \sigma_{-, \downarrow}^{(b)}\right]+A_{\uparrow}^{(c)} \sigma_{+, \downarrow}^{(a) *} \psi_{+, \uparrow}^{(b)}\left[\sigma_{-, \uparrow}^{(a) *} \sigma_{+, \downarrow}^{(a)}\right]\right. \\
& \left.-A_{\downarrow}^{(c)} \sigma_{+, \uparrow}^{(a) *} \psi_{+, \downarrow}^{(b)}\left[\sigma_{-, \downarrow}^{(a) *} \sigma_{+, \uparrow}^{(a)}\right]-A_{\downarrow}^{(c)} \psi_{+, \uparrow}^{(a) *} \sigma_{+, \downarrow}^{(b)}\left[\sigma_{+, \downarrow}^{(b) *} \sigma_{-, \uparrow}^{(b)}\right]\right\} .
\end{aligned}
$$

Similarly, for $I_{1}$ we obtain

$$
\begin{aligned}
I_{1}= & Z_{-} \int d x^{-} d^{2} x^{\perp} \sum_{a}\left\{\left(i \partial_{\uparrow} \psi_{+, \downarrow}^{(a) *}\right) \sigma_{+, \uparrow}^{(a)}\left[\sigma_{+, \uparrow}^{(a) *} \sigma_{-, \downarrow}^{(a)}\right]+\sigma_{+, \downarrow}^{(a) *}\left(i \partial_{\uparrow} \psi_{+, \uparrow}^{(a)}\right)\left[\sigma_{-, \uparrow}^{(a) *} \sigma_{+, \downarrow}^{(a)}\right]\right. \\
& \left.-\sigma_{+, \uparrow}^{(a) *}\left(i \partial_{\downarrow} \psi_{+, \downarrow}^{(a)}\right)\left[\sigma_{-, \downarrow}^{(a) *} \sigma_{+, \uparrow}^{(a)}\right]-\left(i \partial_{\downarrow} \psi_{+, \uparrow}^{(a) *}\right) \sigma_{+, \downarrow}^{(a)}\left[\sigma_{+, \downarrow}^{(a) *} \sigma_{-, \uparrow}^{(a)}\right]\right\} .
\end{aligned}
$$

We are now in a position to explain why the eigenvalue problem (21) can be reformulated with the trivial vacuum. The naive light-cone physical subspace consists of all polynomials in $\psi_{+}$and $A_{\perp}$ applied to the light-cone bare vacuum $|0\rangle$. We shall refer to this type of operator $\mathcal{O}_{P}$ as a physical operator and label the subspace of vectors $\mathcal{O}_{P}|0\rangle$ as $S_{0}$. The operators that will create physical states from the dressed vacuum are just these physical operators, $|\Psi\rangle=\mathcal{O}_{P}|\Omega\rangle . P^{-}=P_{P}^{-}+I+G_{0}$ will be the sum of a part, $P_{P}^{-}$, consisting entirely of physical operators, plus $I$, plus any other terms involving interactions induced by gluon 
modes in the vacuum. Here we will set $G_{0}$ equal to zero and discuss the eigenvalue problem that results from including only the physical operators and $I . P^{+}$and $P^{\perp}$ are physical operators and are kinematical, so we may freely choose their value when we formulate the problem. We use

$$
P^{\perp}=0 \quad, \quad P^{+}=1
$$

The eigenvalue equation (21) then takes the form

$$
\left(P_{P}^{-}+I\right) \mathcal{O}_{P}|\Omega\rangle=M^{2} \mathcal{O}_{P}|\Omega\rangle
$$

From (27) (28), we see that $I$ can be written in the general form

$$
I \sim I_{P}\left[\sigma_{ \pm}^{*} \sigma_{\mp}\right]
$$

where $I_{P}$ is a physical operator and $\left[\sigma_{ \pm}^{*} \sigma_{\mp}\right]$ is a bilinear form occurring in $\left|\Omega_{f}\right\rangle$. Suppose $\mathcal{P}$ projects onto $S_{0}$. Any physical operator commutes with $\mathcal{P}$. From the form given in (19), we see that any component of $|\Omega\rangle$, other than the bare vacuum $|0\rangle$, must contain quanta from the $\psi_{-}^{0}$ field or gluon zero modes. Therefore, $\mathcal{P}|\Omega\rangle=c|0\rangle$ for some constant $c$. We also see that if an operator of the form $\sigma_{-, s}^{(a) *}\left(x_{\perp}\right) \sigma_{+,-s}^{(a)}\left(x_{\perp}\right)$ or its conjugate acts on a component of $\left|\Omega_{f}\right\rangle$ in such a way as to remove all the $\sigma_{-}$spurions, then it must also remove all the $\sigma_{+}$ spurions. Therefore $\mathcal{P} \sigma_{-, s}^{(a) *}\left(x_{\perp}\right) \sigma_{+,-s}^{(a)}\left(x_{\perp}\right)|\Omega\rangle=\kappa|0\rangle$, where $\kappa$ is a real constant independent of $s, a$, and $x_{\perp}$, as follows from Poincare and gauge invariance.

If we now operate on both sides of equation (30) with $\mathcal{P}$ and divide through by $c$, we obtain

$$
\left(P_{P}^{-}+I_{P} \frac{\kappa}{c}\right) \mathcal{O}_{P}|0\rangle=M^{2} \mathcal{O}_{P}|0\rangle
$$

This equation has the same spectrum as (21) and the eigenvectors of (32) are equal to the projections of the eigenvectors of (21) onto $S_{0}$. Equation (32) is an equation in the naive lightcone subspace. Of course, details of the dressed vacuum $|\Omega\rangle$ have been swept into the unknown constants $\kappa$ and $c$. In the following, we analyze the details of the action of $I_{P}$ on physical states, showing explicitly how it violates quark helicity.

For the projected equation (32), the operators in $I_{P}$ are given by (27) (28) without the spurions in square brackets. In order to demonstrate the action of $I_{P}$ on states in $S_{0}$, at this point it is necessary to reintroduce the system in the box of Fig.1. This will also facilitate later numerical work, where periodicity for bosons and anti-periodicity for fermions over 
$x^{-} \rightarrow x^{-}+2 L$ introduces a DLCQ 20] harmonic resolution cutoff $K=L / \pi$. Any state in $S_{0}$ can be written schematically (suppressing all arguments) as

$$
\sum_{i=1}^{\infty} \sum_{j=0}^{\infty} \sum_{k=0}^{\infty} f_{i, j, k}\left(b^{*}\right)^{i}\left(d^{*}\right)^{j}\left(a^{*}\right)^{k}|0\rangle
$$

To operate on such a state we can use the following properties of the spurions [17]

$$
\begin{aligned}
& \sigma_{+, s}^{(a) *}|0\rangle=\mathrm{e}^{\frac{\mathrm{i} x^{-}}{2 K}} b_{s}^{(a) *}(1 / 2 K)|0\rangle \\
& \sigma_{+, s}^{(a)}|0\rangle=\mathrm{e}^{\frac{\mathrm{i} x^{-}}{2 K}} d_{-s}^{(a) *}(1 / 2 K)|0\rangle
\end{aligned}
$$

which converts them to $\psi_{+}$modes of the lowest allowed lightcone momentum in DLCQ, $1 / 2 K$. Note that this shows that $\sigma_{+}^{*}$ transforms in the fundamental representation of the residual gauge group in lightcone gauge when it acts on $|0\rangle$. The following relations are also useful: unless $s=s^{\prime} ; a=b$ and $x_{\perp}=y_{\perp}\left\{\sigma_{+, s}^{(a)}\left(x_{\perp}\right), b_{s^{\prime}}^{(b) *}\left(k^{+}, y_{\perp}\right)\right.$ or $\left.d_{-s^{\prime}}^{(b) *}\left(k^{+}, y_{\perp}\right)\right\}=0$; otherwise

$$
\begin{aligned}
& \sigma_{+, s}^{(a)} b_{s}^{(a) *}((2 n-1) / 2 K)=\mathrm{e}^{\frac{-\mathrm{i} x^{-}}{K}} b_{s}^{(a) *}((2 n-3) / K) \sigma_{+, s}^{(a)} \\
& \sigma_{+, s}^{(a)} d_{-s}^{(a) *}((2 n-1) / 2 K)=\mathrm{e}^{\frac{\mathrm{i} x^{-}}{K}} d_{-s}^{(a) *}((2 n+1) / 2 K) \sigma_{+, s}^{(a)} \\
& \sigma_{+, s}^{(a) *} b_{s}^{(a) *}((2 n-1) / 2 K)=\mathrm{e}^{\frac{\mathrm{i} x^{-}}{K}} b_{s}^{(a) *}((2 n+1) / 2 K) \sigma_{+, s}^{(a) *} \\
& \sigma_{+, s}^{(a) *} d_{-s}^{(a) *}((2 n-1) / 2 K)=\mathrm{e}^{\frac{-\mathrm{i} x^{-}}{K}} d_{-s}^{(a) *}((2 n-3) / 2 K) \sigma_{+, s}^{(a) *},
\end{aligned}
$$

except that

$$
\begin{aligned}
& \sigma_{+, s}^{(a)} b_{s}^{(a) *}(1 / 2 K)=\mathrm{e}^{\frac{-\mathrm{i} x^{-}}{K}} d_{-s}^{(a)}(1 / 2 K) \sigma_{+, s}^{(a)} \\
& \sigma_{+, s}^{(a) *} d_{-s}^{(a) *}(1 / 2 K)=\mathrm{e}^{\frac{-\mathrm{i} x^{-}}{K}} b_{s}^{(a)}(1 / 2 K) \sigma_{+, s}^{(a) *} .
\end{aligned}
$$

If we apply $I_{P}$ from $I_{2}$ (27) to a quark or antiquark in the state (33), we obtain

$$
\begin{aligned}
& I_{P} b_{s}^{(a) *}(x+1 / 2 K)|0\rangle=s g Z_{-} \sqrt{\frac{\pi}{x}} \sum_{b c} \lambda_{a b}^{c} b_{-s}^{(b) *}(1 / 2 K) a_{s}^{(c) *}(x)|0\rangle \\
& I_{P} d_{s}^{(b) *}(x+1 / 2 K)|0\rangle=-s g Z_{-} \sqrt{\frac{\pi}{x}} \sum_{b c} \lambda_{a b}^{c} d_{-s}^{(a) *}(1 / 2 K) a_{s}^{(c) *}(x)|0\rangle
\end{aligned}
$$

where $s=+1 \Longrightarrow \uparrow, s=-1 \Longrightarrow \downarrow$, and $x$ is a positive integer multiple of $1 / K$. The interaction puts the quark in the lowest DLCQ lightcone momentum state and flips its helicity. The same operator also produces pair annihilation:

$$
\begin{aligned}
I_{P} b_{s}^{(a) *}(x-1 / 2 K) d_{s}^{(b) *}(1 / 2 K)|0\rangle & =I_{P} b_{s}^{(a) *}(1 / 2 K) d_{s}^{(b) *}(x-1 / 2 K)|0\rangle \\
& =-s g Z_{-} \sqrt{\frac{\pi}{x}} \sum_{c} \lambda_{a b}^{c} a_{s}^{(c) *}(x)|0\rangle .
\end{aligned}
$$


Notice that the pair destruction only occurs when one quark is in the lowest DLCQ lightcone momentum state, so pair production always creates such a state. If we apply $I_{P}$ from $I_{1}$ (28) to a quark or antiquark in the state (33), we find that it only acts on particles already in the lowest DLCQ lightcone momentum state. In this case, we obtain

$$
\begin{aligned}
& I_{P} b_{s}^{(a) *}\left(1 / 2 K, x_{\perp}\right)|0\rangle=i s Z_{-} \partial_{s} b_{-s}^{(a) *}\left(1 / 2 K, x_{\perp}\right)|0\rangle \\
& I_{P} d_{s}^{(a) *}\left(1 / 2 K, x_{\perp}\right)|0\rangle=-i s Z_{-} \partial_{s} d_{-s}^{(a) *}\left(1 / 2 K, x_{\perp}\right)|0\rangle
\end{aligned}
$$

The interaction flips the helicity of this quark while changing the 'orbital' angular momentum of the state to keep angular momentum projection $J_{3}$ conserved.

Let us summarize the rather technical analysis of this section. The net effect of the induced operator $I$ is in all cases to flip the helicity of a quark or anti-quark. Angular momentum is conserved either by the emission of a gluon, leaving the quark in the lowest DLCQ momentum state, or a change in the orbital angular momentum of a quark already in the lowest DLCQ momentum state. Thus, quark helicity is no longer conserved. The induced operator can also cause pair production of same-helicity quarks and antiquarks when one of them is in the lowest allowed DLCQ momentum state. These processes are illustrated in Figure 2, where we defined the effective coupling constant

$$
h=\frac{g Z_{-} \kappa}{c} .
$$

Evidently, all of the previous discussion goes through with any number of spectator gluons and quarks in a hadron state. The non-conservation of quark helicity is crucial to obtaining a splitting of masses between pseudoscalar and vector mesons. In the absence of $I$ they would be degenerate. We mention that in a $U(N)$ gauge theory the spin exchange in the valence state can proceed by annihilation through the color diagonal component of the gauge field. In the case of QED, for example, this contributes to the hyperfine splitting of positronium. In a chiral $S U(N)$ gauge theory, spontaneous (or anomalous) non-conservation of quark helicity is the only way to achieve it. 

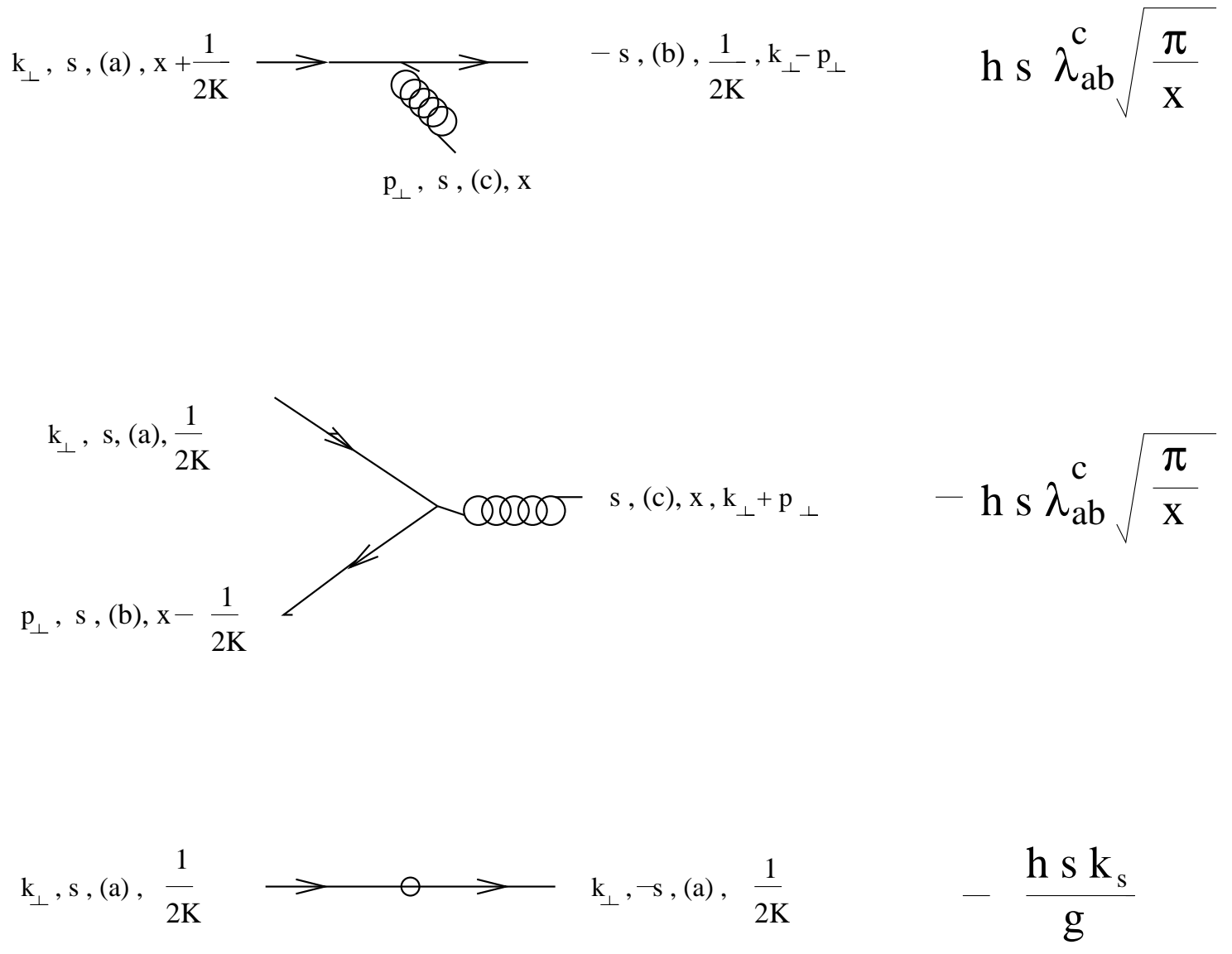

FIG. 2: The elementary vertices of lightcone perturbation theory in momentum space, resulting from the induced operator $I$. They show the change of helicity $s$, lightcone momentum $x$, colour $(a)$, and transverse momentum $k_{\perp}$, where $k_{s}$ is the eigenvalue of $-i \partial_{s}, s \in\{\uparrow, \downarrow\} .1 / 2 K$ is the smallest unit of fermion lightcone momentum in DLCQ with cutoff $K . h$ is the combination of gauge coupling and vacuum coefficients defined in eq.(47). The same vertices apply for anti-quarks with arrows on quark lines reversed but with an extra overall minus sign for the first and third amplitude (see eqs. (43) (46)).

\section{SOLUTIONS FOR DIMENSIONALLY REDUCED MODELS}

\section{A. Overview}

In this section, we look at meson solutions for simple dimensionally reduced models in $1+1$ dimensions obtained by restricting to the $k_{\perp}=0$ sector, meaning that we (classically) 
discard all fields except the transverse zero modes satisfying

$$
\partial_{x_{\perp}} A_{\mu}=\partial_{x_{\perp}} \psi=0
$$

These calculations, although not physically realistic, are straightforward enough to let us illustrate explicitly the effects on the meson spectrum alluded to above and also allow us to investigate how things might scale with the DLCQ cutoff. In the present subsection, we find exact solutions for the dimensionally reduced lightcone hamiltonian containing only the induced operator $I$. In the next subsections we analyze a slightly more realistic large- $N$ gauged version of these dimensionally reduced models. In both cases, $I$ obviously consists of the part $I_{2}(27)$ only.

The dimensionally reduced theories inherit various symmetries from the $3+1$ dimensions. They preserve total quark and gluon helicity, identified with the angular momentum projection $J_{3}$ for physical operators, a remnant of the $3+1$ dimensional rotation symmetry. This automatically leads to doublets in the spectrum consisting of opposite helicities. There is also exact charge conjugation symmetry $C$ and an exact kinematic parity symmetry $P$ of the valence part of wavefunctions: $f^{2}(x, 1-x) \leftrightarrow f^{2}(1-x, x)$, where $x$ is the quark lightcone momentum and $1-x$ the antiquark momentum. This kinematic valence parity only equals parity of the full wavefunction in the free field limit, but is a convenient label. Thus, bound states can be labelled by $\left|J_{3}\right|^{P C}$. We will be particularly be interested in the quantum numbers $0^{- \pm}$and $1^{--}$, which together form the quantum numbers of a pseudoscalar, such as the $\pi$ or $\eta^{\prime}$, and the three Lorentz components of a vector, such as the $\rho$ or $\phi-\omega$ meson. Generically, the $1^{--}$doublet and the $0^{--}$are split because of dimensional reduction. We do not address the issue here of how to make a full degenerate 3+1-dimensional Lorentz multiplet for the vector, as this really requires transverse motion. Rather, we wish to study splitting of the $0^{- \pm}$states, since this occurs only if the quark helicity is not conserved. In $1+1$ dimensions, to have canonically normalised kinetic terms, we rescale fields $\psi \rightarrow \psi \sqrt{V_{\perp}}$, $A_{\perp} \rightarrow A_{\perp} \sqrt{V_{\perp}}$, where $V_{\perp}=\int d x^{1} d x^{2}$ is the transverse volume factor. The gauge coupling also becomes dimensionful through dimensional reduction, $g^{2} \rightarrow g^{2} V_{\perp} N$, where we have also absorbed the colour factor $N$ in the large $N$ limit. 


\section{B. I only}

In this subsection we shall consider the case where the entire lightcone hamiltonian $P^{-}$ in the projected subspace $S_{0}$ is given by $I$ (23). In this case the colour label is rather redundant, so we omit it. We can expand the wave function of a helicity-zero 'meson' as

$$
\begin{aligned}
|\phi\rangle & =\sum_{x} f_{\uparrow \downarrow}^{2}(x) b_{\uparrow}^{*}(x) d_{\downarrow}^{*}(1-x)|0\rangle \\
& +\sum_{x+y \leq 1} f_{\downarrow \uparrow \downarrow}^{3}(x, y) b_{\downarrow}^{*}(x) d_{\downarrow}^{*}(1-x-y) a_{\uparrow}^{*}(y)|0\rangle \\
& +\sum_{x+y+z \leq 1} f_{\uparrow \downarrow \uparrow \downarrow}^{4}(z, x, y) b_{\uparrow}^{*}(z) d_{\downarrow}^{*}(1-x-y-z) a_{\downarrow}^{*}(x) a_{\uparrow}^{*}(y)|0\rangle \\
& +[\downarrow \leftrightarrow \uparrow]
\end{aligned}
$$

This expansion includes all the states which can couple to the 'valence' $b d$ sector in this model, so it is a complete expansion of the wave function in the helicity zero sector. The eigenvalue equation (32), when projected onto specific Fock states in the expansion (49), leads to the following boundstate equations for the meson invariant mass $M$

$$
\begin{aligned}
M^{2} f_{\uparrow \downarrow}^{2}(x) & =\frac{h}{\sqrt{x}} f_{\downarrow \uparrow \downarrow}^{3}(0, x) \\
& +\frac{h}{\sqrt{1-x}} f_{\uparrow \downarrow \uparrow}^{3}(x, 1-x) \\
M^{2} f_{\downarrow \uparrow \downarrow}^{3}(x, y)= & \frac{h}{\sqrt{y}} f_{\uparrow \downarrow}^{2}(x+y) \delta(x) \\
- & \frac{h}{\sqrt{y}} f_{\uparrow \downarrow}^{2}(x) \delta(1-x-y) \\
- & \frac{h}{\sqrt{x}} f_{\uparrow \downarrow \uparrow \downarrow}^{4}(0, x, y) \\
+ & \frac{h}{\sqrt{1-x-y}} f_{\downarrow \uparrow \downarrow \uparrow}^{4}(x, y, 1-x-y) \\
M^{2} f_{\uparrow \downarrow \uparrow \downarrow}^{4}(z, x, y) & =-\frac{h}{\sqrt{x}} f_{\downarrow \uparrow \downarrow}^{3}(z+x, y) \delta(z) \\
& -\frac{h}{\sqrt{y}} f_{\uparrow \downarrow \uparrow}^{3}(z, x) \delta(1-y-z-x)
\end{aligned}
$$

with the same equations with $\uparrow \leftrightarrow \downarrow, h \leftrightarrow-h$. 0 is a shorthand for $1 / 2 K$, and 1 for $1-1 / 2 K$; if a denominator vanishes, then that term is excluded from the equation; we define $\delta(x)=0$ if $x \neq 0$ otherwise 1 . This simple model is interesting only because the equations can be 
solved algebraically. They lead to the following effective equations in the valence sector.

$$
\begin{aligned}
M^{2} f_{\uparrow \downarrow}^{2}(0) & =\frac{h^{2}}{M^{2}}\left(f_{\uparrow \downarrow}^{2}(0)-f_{\downarrow \uparrow}^{2}(1)\right) \\
\left.M^{2} f_{\uparrow \downarrow}^{2}(x)\right|_{x \neq 0,1} & =\left.\frac{h^{2}}{M^{2} x(1-x)}\left(1+\frac{4}{\frac{M^{4}}{h^{2}}-\frac{1}{x(1-x)}}\right) f_{\uparrow \downarrow}^{2}(x)\right|_{x \neq 0,1}
\end{aligned}
$$

Solutions of the first of these equations for $f$ are delta functions at 0,1 and give the following results for the $0^{-+}$state

$$
f_{\uparrow \downarrow}(1)=f_{\uparrow \downarrow}(0) ; \quad f_{\uparrow \downarrow}=-f_{\downarrow \uparrow} ; \quad M^{2}= \pm \sqrt{2} h
$$

and $0^{--}$state

$$
f_{\uparrow \downarrow}(1)=f_{\uparrow \downarrow}(0) ; \quad f_{\uparrow \downarrow}=f_{\downarrow \uparrow} ; \quad M^{2}=0 .
$$

The masses are split even in the DLCQ continuum limit $K \rightarrow \infty$.

There are a number of artifacts that occur due to keeping only $I$ in this simple model. The lowest lying states are tachyonic. Opposite parity states $\left(f_{\uparrow \downarrow}(1)= \pm f_{\uparrow \downarrow}(0)\right)$ are degenerate. Also, there are solutions to eq.(154) which are delta functions at specific values $0<x<1 / 2$ with

$$
M^{4}=h^{2} \frac{1 \pm 2 \sqrt{x(1-x)}}{x(1-x)} .
$$

This spectrum is continuous and, given that both signs are possible for $M^{2}$, unbounded below in the DLCQ continuum limit $K \rightarrow \infty$. The unbound solutions at small $x>1 / 2 K$ are an artifact due to wee gluon emission. These artifacts will be avoided in the more realistic gauged model.

A similar analysis for the $1^{--}$sector produces $M^{4}=h^{2}$ for delta function wavefunctions at $x=0,1$. These would partner the $0^{--}$state and, although they are not degenerate in the reduced model, would eventually form the vector Lorentz multiplet in higher dimensions.

\section{Dimensionally reduced gauge theory}

Since the quark helicity violating term $I$ couples to the quark of miminal DLCQ momentum only, one might worry that the effects shown in the last section disappear in the DLCQ continuum limit $L \rightarrow \infty$ of a non-trivial theory that has wavefunctions with support on all momenta. We demonstrate in the following with explicit numerical DLCQ calculations that this is not the case. In this section, to be consistent with previous literature, we use the 
convention $x^{ \pm}=\left(x^{0} \pm x^{3}\right) / \sqrt{2}$. The light-cone hamiltonian we use starts from the large $N$ generalisation of eq. (11), dimensionally reduced to $1+1$ dimensions, with appropriately rescaled fields and coupling. This results in a reduced action that was first studied in ref. 21]

$$
\begin{aligned}
S \rightarrow & \int d x^{+} d x^{-}\left\{-\frac{1}{4} \operatorname{Tr} F_{\alpha \beta} F^{\alpha \beta}+\frac{\mathrm{i}}{\sqrt{2}}\left(\bar{u}_{\uparrow} \gamma_{(2)}^{\alpha} D_{\alpha} u_{\uparrow}+\bar{u}_{\downarrow} \gamma_{(2)}^{\alpha} D_{\alpha} u_{\downarrow}\right)\right. \\
& +\operatorname{Tr}\left[-\frac{1}{2} \bar{D}_{\alpha} A_{r} \bar{D}^{\alpha} A^{r}-\frac{g^{2}}{4 N}\left[A_{r}, A_{s}\right]\left[A^{r}, A^{s}\right]+\frac{1}{2} m_{0}^{2} A_{r} A^{r}\right] \\
& \left.-\frac{g}{\sqrt{2 N}}\left(\bar{u}_{\uparrow}\left(A_{1}+\mathrm{i} \gamma_{(2)}^{5} A_{2}\right) u_{\uparrow}-\bar{u}_{\downarrow}\left(A_{1}-\mathrm{i} \gamma_{(2)}^{5} A_{2}\right) u_{\downarrow}\right)\right\} .
\end{aligned}
$$

$\alpha$ and $\beta \in\{+,-\}, r, s \in\{1,2\}, \gamma_{(2)}^{0}=\sigma^{1}, \gamma_{(2)}^{3}=\mathrm{i} \sigma^{2}, \gamma_{(2)}^{5}=\mathrm{i} \sigma^{1} \sigma^{2}, \bar{D}_{\alpha}=\partial_{\alpha}+\mathrm{i} g\left[A_{\alpha},.\right] / \sqrt{N}$, $D_{\alpha}=\partial_{\alpha}+\mathrm{i} g A_{\alpha} / \sqrt{N}$. The two-component spinors $u_{\uparrow}$ and $u_{\downarrow}$ are related to the original $3+1$ dimensional $\psi$ field by

$$
2^{1 / 4} \psi \sqrt{\int d x^{1} d x^{2}}=\left(\begin{array}{c}
u_{+, \uparrow} \\
u_{-, \uparrow} \\
u_{-, \downarrow} \\
u_{+, \downarrow}
\end{array}\right), u_{\uparrow}=\left(\begin{array}{c}
u_{-, \uparrow} \\
u_{+, \uparrow}
\end{array}\right), u_{\downarrow}=\left(\begin{array}{c}
u_{-, \downarrow} \\
u_{+, \downarrow}
\end{array}\right) .
$$

Since the gluon mass is not protected by transverse gauge symmetry transformations in a dimensionally reduced model, we must allow a gluon mass $m_{0}$ in general. In fact, this will regulate small- $x$ gluon divergences. $u_{\uparrow}, u_{\downarrow}, A_{1}, A_{2}$ represent the transverse polarizations of the $3+1$ dimensional quarks and gluons. In $1+1$ dimensions, where there is of course no spin, the fields appear as different 'flavours' in fundamental and adjoint representations.

The $u_{\uparrow}$ and $u_{\downarrow}$ fields in (58) have separate conserved $U(1)$ fermion numbers, but no axial symmetries $\left(\right.$ with $\left.\gamma_{(2)}^{5}\right)$. This $U(1) \times U(1)$ transcribes to the left and right handed $U(1)$ symmetries in $3+1$ dimensions of a single flavour of massless quarks in QCD. Thus, the dimensionally reduced model has the important property that it inherits the chiral symmetries of QCD with massless quarks. Note that, with a single flavour of quarks, the axial anomaly may spoil one of the $U(1)$ symmetries, but in the large $N$ limit the anomaly is suppressed as it involves fermion pair production. It is also necessary to work at large- $N$ to have spontaneous symmetry breaking of continuous symmetry in $1+1$ dimensions. It was shown in ref. 22], by completely different methods, that this reduced theory has spontaneous 
$U(1)$ symmetry breaking. The analogy with $3+1$ dimensions at large $N$ and with one flavour, is that one expects the axial $U(1)_{A}$ combination to be spontaneously broken. In the reduced model, this corresponds to the $U(1) \times U(1)$ flavour symmetry being broken down to its diagonal 'total' fermion number subgroup. The broken $U(1)$ corresponds to the charge measuring quark helicity. Without the dimensionally reduced version of the induced operator $I$, the hamiltonian contains no terms that flip quark helicity. In that case, with a trivial vacuum, we find that the states $0^{- \pm}$are degenerate. In the following, we add $I$ to the calculation in the spirit of the last section, assuming there is a non-zero condensate from the outset.

\section{Boundstate Equations}

In the light-cone gauge, the fields $A_{+}$and non-zero modes of $u_{-}$are non-propagating in light-front time $x^{+}=\left(x^{0}+x^{3}\right) / \sqrt{2}$. We eliminate them using their constraint equations of motion. The expansion in creation and annihilation operators for the dimensionally reduced fermion $u_{+, s}^{(a)}$ and gluon $A_{s}^{(c)}$ follows eqs. (6) (19) with transverse momentum dependence dropped. For a helicity-zero meson in the projected subspace $S_{0}$, the state invariant under residual gauge transformations is written

$$
\begin{aligned}
& \sum_{a} \frac{1}{N} \int d x f_{\uparrow \downarrow}^{2}(x, 1-x) b_{\uparrow}^{(a) *}(x) d_{\downarrow}^{(a) *}(1-x)+\int d x f_{\downarrow \uparrow}^{2}(x, 1-x) b_{\downarrow}^{(a) *}(x) d_{\uparrow}^{(a) *}(1-x) \\
& +\sum_{a b c} \frac{1}{N^{2}} \int d x d y f_{\downarrow \uparrow \downarrow}^{3}(x, y, 1-x-y) b_{\downarrow}^{(a) *}(x) \lambda_{a b}^{c} a_{\uparrow}^{(c) *}(y) d_{\downarrow}^{(b) *}(1-x-y)+ \\
& \frac{1}{N^{2}} \int d x d y f_{\uparrow \downarrow \uparrow}^{3}(x, y, 1-x-y) b_{\uparrow}^{(a) *}(x) \lambda_{a b}^{c} a_{\downarrow}^{(c) *}(y) d_{\uparrow}^{(b) *}(1-x-y)+\cdots|0\rangle
\end{aligned}
$$

where $\cdots$ indicates higher numbers of gluon creation operators $a^{*}$. The wavefunction components are normalised as

$$
\begin{aligned}
& \int_{0}^{1} d x\left|f_{\uparrow \downarrow}^{2}(x, 1-x)\right|^{2}+\left|f_{\downarrow \uparrow}^{2}(x, 1-x)\right|^{2} \\
& +\int_{0}^{1} d x \int_{0}^{1-x} d y\left|f_{\downarrow \uparrow \downarrow}^{3}(x, y, 1-x-y)\right|^{2}+\left|f_{\uparrow \downarrow \uparrow}^{3}(x, y, 1-x-y)\right|^{2}+\cdots=1
\end{aligned}
$$

As before, boundstate equations for the wavefunctions $f$ are obtained by applying the lightcone hamiltonian $P^{-}$to a meson state, such as eq.(60), and then projecting onto a given vector in the physical Fock space. If we neglect contributions from gluon zero modes, 
the resulting equations are the same as those given in ref. 21], plus the helicity-violating induced interaction $I$. Since we will solve these equations numerically in section IVE, in a truncation of the Fock space to the sectors of $f^{2}$ and $f^{3}$ (one-gluon approximation), we display only the equations for this truncation:

$$
\begin{aligned}
M^{2} f_{\uparrow \downarrow}^{2}(x, 1-x) & =\frac{m_{f}^{2}}{x(1-x)} f_{+-}^{2}(x, 1-x) \\
& +\frac{g^{2}}{\pi} \int_{0}^{1} d y\left\{\frac{f_{\uparrow \downarrow}^{2}(x, 1-x)-f_{\uparrow \downarrow}^{2}(y, 1-y)}{(y-x)^{2}}\right\} \\
& +\frac{h}{\sqrt{x}} f_{\downarrow \uparrow \downarrow}^{3}(0, x, 1-x) \\
& +\frac{h}{\sqrt{1-x}} f_{\uparrow \downarrow \uparrow}^{3}(x, 1-x, 0)
\end{aligned}
$$

$$
\begin{aligned}
& M^{2} f_{\downarrow \uparrow \downarrow}^{3}(x, y, 1-x-y)=\frac{m_{b}^{2}}{y} f_{\downarrow \uparrow \downarrow}^{3}(x, y, 1-x-y) \\
& +\frac{g^{2}}{\pi} \int_{0}^{1-x} d z \frac{1+y-x-z}{2(1-x-y-z)^{2} \sqrt{y(1-x-z)}}\left\{f_{\downarrow \uparrow \downarrow}^{3}(x, y, 1-x-y)\right. \\
& \left.-f_{\downarrow \uparrow \downarrow}^{3}(x, 1-x-z, z)\right\} \\
& +\frac{g^{2}}{\pi(1-x-y)}\left(\sqrt{1+\frac{1-x-y}{y}}-1\right) f_{\downarrow \uparrow \downarrow}^{3}(x, y, 1-x-y) \\
& +\frac{g^{2}}{\pi} \int_{0}^{x+y} d z \frac{x+2 y-z}{2(x-z)^{2} \sqrt{y(x+y-z)}}\left\{f_{\downarrow \uparrow \downarrow}^{3}(x, y, 1-x-y)\right. \\
& \left.-f_{\downarrow \uparrow \downarrow}^{3}(z, x+y-z, 1-x-y)\right\} \\
& +\frac{g^{2}}{\pi x}\left(\sqrt{1+\frac{x}{y}}-1\right) f_{\downarrow \uparrow \downarrow}^{3}(x, y, 1-x-y) \\
& +\frac{g^{2}}{\pi} \int_{0}^{1-x} d z \frac{f_{\downarrow \uparrow \downarrow}^{3}(x, z, 1-x-z)}{(1-x) \sqrt{y z}} \\
& +\frac{g^{2}}{\pi} \int_{0}^{x+y} d z \frac{f_{\downarrow \uparrow \downarrow}^{3}(x+y-z, z, 1-x-y)}{(x+y) \sqrt{y z}} \\
& +\frac{h}{\sqrt{y}} f_{\uparrow \downarrow}^{2}(x+y, 1-x-y) \delta(x) \\
& -\frac{h}{\sqrt{y}} f_{\downarrow \uparrow}^{2}(x, 1-x) \delta(1-x-y)
\end{aligned}
$$

and the same equations with $\uparrow \leftrightarrow \downarrow, h \leftrightarrow-h$. Note that $M^{2}$ is the eigenvalue of $2 P^{-}$is the normalisation of this subsection. $m_{b}$ is the gluon mass $m_{0}$ after renormalisation resulting from normal ordering of gluon fields in $P^{-}$. Although not necessary for our purposes of demonstrating pseudoscalar-vector splitting, for generality we added a quark 'kinetic' mass 


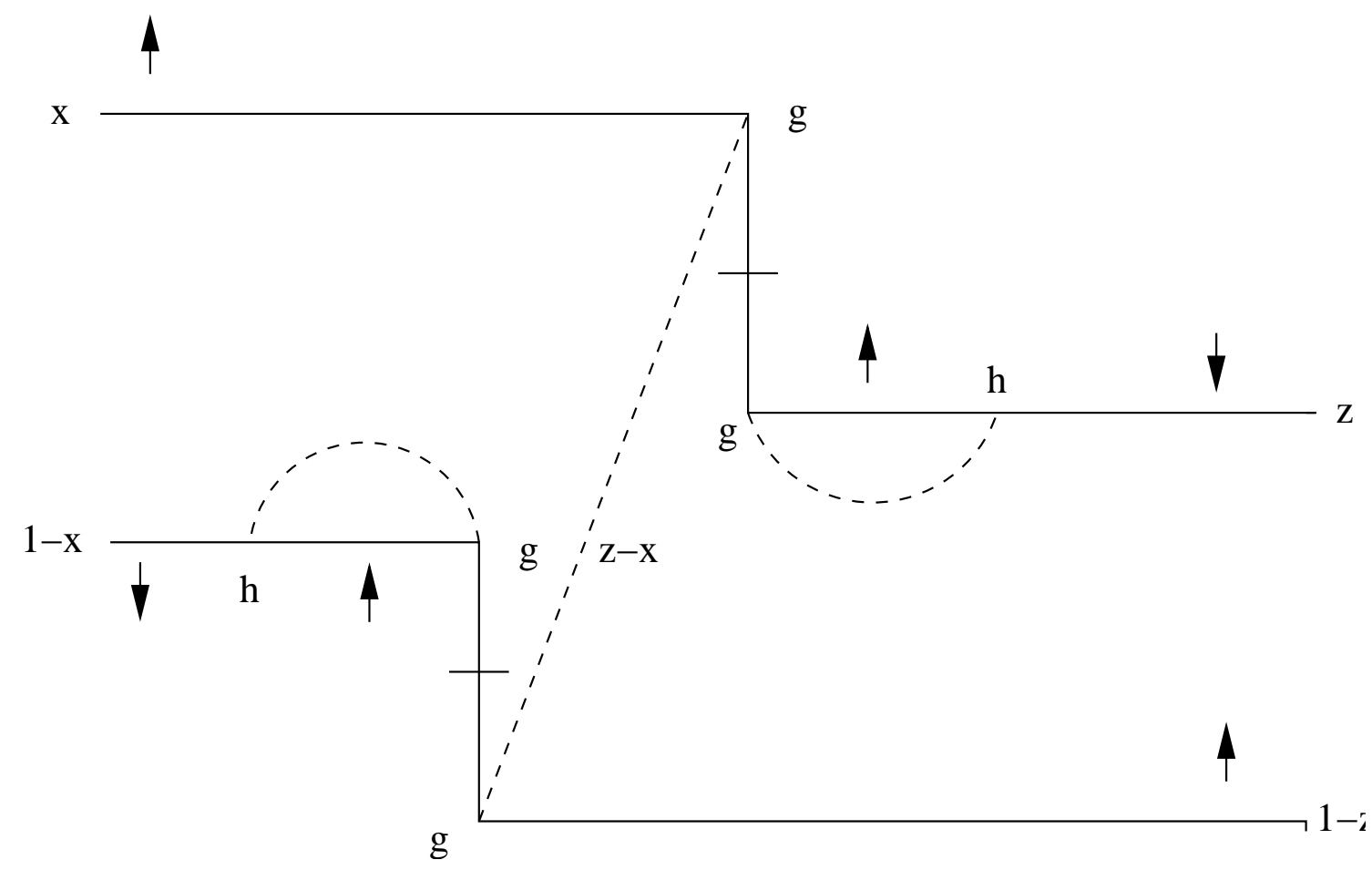

FIG. 3: Quark helicity flip process involving $I$. Solid lines are quarks $\left(x^{+}\right.$-instantaneous when barred), chain lines are dimensionally reduced gluons. Vertices are labelled by their corresponding couplings.

$m_{f}$ in the $f^{2}$ sector. Such counterterms in the hamiltonian should be allowed since DLCQ and a one-gluon truncation break symmetries such as parity [23]. Most importantly for us, a quark kinetic mass term does not break quark helicity symmetry.

\section{E. DLCQ gauge theory solution}

The endpoint delta functions of momentum, seen in section IVB now become spread out by the additional interactions in the gauge theory. The $I$ interaction, that acts at endpoints only, couples to a part of the wavefunction of measure zero. One therefore expects the direct effects of $I$ in splitting the $0^{-+}$and $0^{--}$to vanish as DLCQ $K \rightarrow \infty$. However, $I$ can combine with other interactions to produce helicity-flip effects away from the endpoints.

An example at order $h^{2} g^{4}$ is illustrated in Figure 3. The value that this process contributes to the expectation value of $M^{2}$ can be calculated in light-cone perturbation theory:

$$
h^{2}\left(\frac{g^{2}}{\pi}\right)^{2} \int_{0}^{1} d x \int_{x}^{1} d z \frac{f_{\uparrow \downarrow}^{2}(x, 1-x) f_{\downarrow \uparrow}^{2}(z, 1-z)}{(1-x)^{2}\left(M^{2}-\frac{m_{b}^{2}}{1-x}\right)(z-x)\left(M^{2}-\frac{m_{b}^{2}}{z-x}\right)\left(M^{2}-\frac{m_{b}^{2}}{z}\right) z^{2}}
$$


If $f^{2}$ and $M^{2}$ are finite, then this contribution is finite for finite $h$.

We solved numerically in DLCQ the dimensionally reduced QCD boundstate equations truncated to at most one gluon for the range $K=5$ to $K=30$. The particular choice of the parameters $m_{b}, m_{f}, g, h$ is not very important, since we did not try to tune them to obtain the best phenomenology. However, they were zoned to ensure absence of tachyons, that the $0^{- \pm}$states were light in the spectrum, and a reasonably large splitting of the $0^{- \pm}$ states compared to their masses. A typical result for the spectrum in this case is shown in Figure 4, where $M^{2}$ eigenvalues have been fit to the form

$$
M^{2}=A+\frac{B}{\sqrt{K}}+\frac{C}{K}+\frac{D}{K^{2}} .
$$

The graph shows that the splitting of the $0^{- \pm}$survives the DLCQ continuum limit $K \rightarrow \infty$, as advertised.

\section{CONCLUSIONS}

We have shown that operator-valued integration constants, which arise in the solutions of the constraint equations encountered in quantizing QCD in the light-cone representation, can dress the bare light-cone vacuum and induce new interactions. Without specifying the vacuum explicitly, except for the general form following from Poincare and gauge symmetry and the assumption that the bare vacuum is dressed by a pure fermion component, we have derived the form of new operators induced into the lightcone QCD hamiltonian. Those operators violate quark helicity for zero quark bare mass. We have demonstrated qualitatively, and quantitatively in the case of dimensionally reduced models, how that operator leads to a splitting of the masses of the pseudoscalar and vector mesons, the helicity zero components of which would be degenerate otherwise. The induced operators we have derived in this paper are almost certainly not the only ones in QCD. We have not analysed the gluon structure of the vacuum nor the gluon zero mode contribution to the hamiltonian, which is a separate study of interest.

The illustrative DLCQ calculations we carried out for reduced models can in principle be extended to $3+1$ dimensions via, for example, transverse lattices [24, 25] or the Pauli-Villars regulated formulation [26], where similar induced operators will arise to spontaneously break quark helicity symmetry. These topics are now under investigation. Finally we draw the 


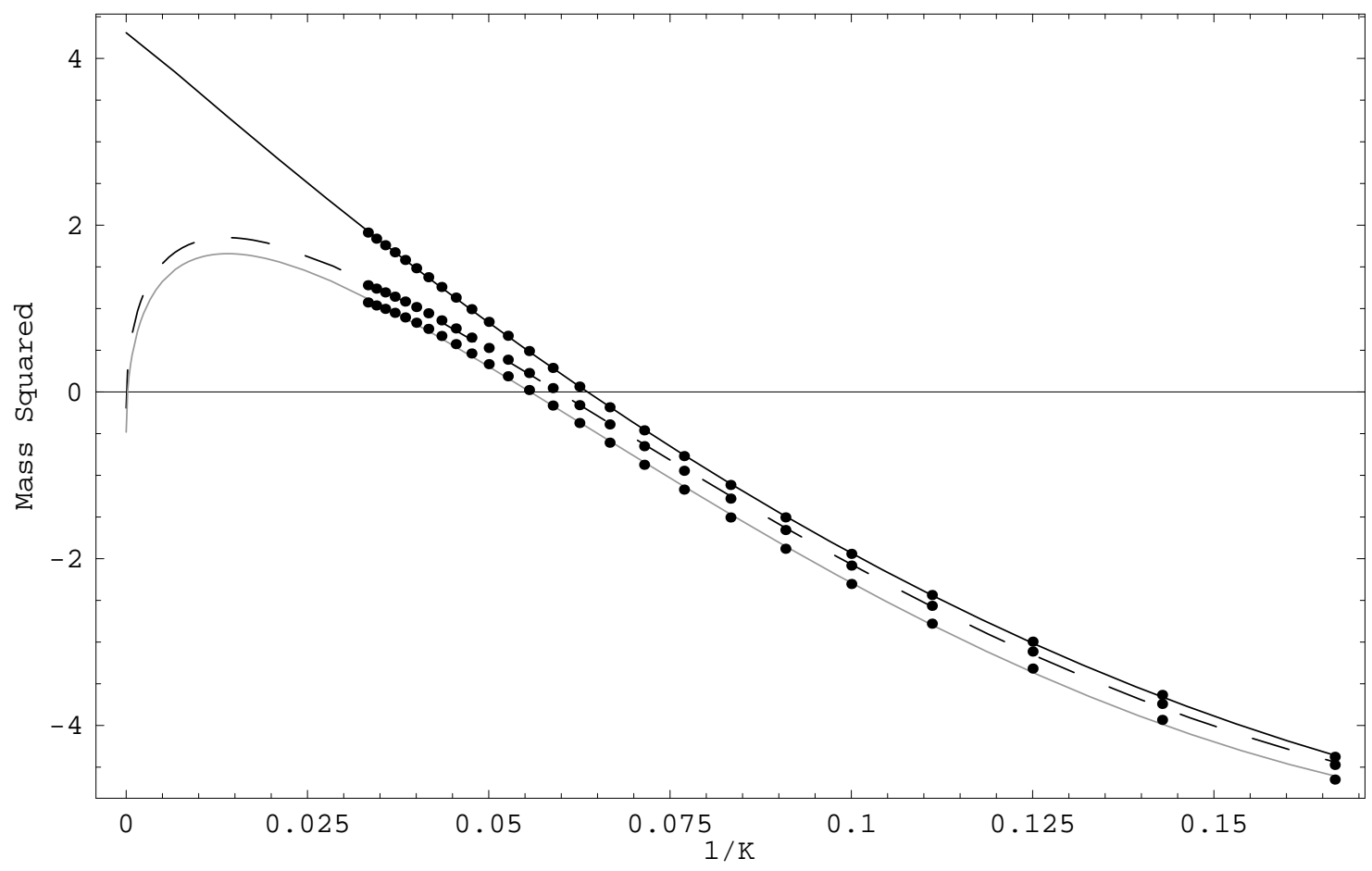

FIG. 4: Variation of mass squared $M^{2}$ with DLCQ cutoff $K$ for the lightest three energy levels. The raw DLCQ data is smoothly extrapolated to $K=\infty$ : dark solid line $0^{--}$; chain line $0^{-+}$. For completeness we show also the $1^{--}$(gray line), which appears anomalously light for this particular parameter choice. 
readers' attention to other recent work on chiral symmetry in light-cone field theory using path integrals [27] and importing chiral symmetry breaking from quantization on a space-like surface by rotating to the characteristic after having analyzed the chiral symmetry breaking [28].

\section{Acknowledgments}

Acknowledgments: The work of SD is supported by PPARC grant PPA/G/0/2002/00470. The work of GM is supported by the U. S. Department of Energy through contract DEFG03-95ER40908. We thank B. van de Sande for helpful discussions.

\section{APPENDIX A: THE SCHWINGER MODEL}

One example for which the vacuum has been derived exactly is the Schwinger model (massless QED in $1+1$ dimensions). There exists a complete operator solution which can be evaluated either at $x^{0}=0$ or at $x^{+}=0$ [4]. Since there is one operator solution, if it has a condensate when quantized at equal time there must also be the same one when quantized on the light cone. Here, we shall simply review those results, referring to the literature [3, 4, 19] for details and derivations. The action is

$$
S=\int d^{2} x\left[-\frac{1}{4} \operatorname{Tr} F_{\alpha \beta} F^{\alpha \beta}+\mathrm{i} \bar{\psi} \gamma_{(2)}^{\alpha} D_{\alpha} \psi\right],
$$

$\alpha, \beta \in\{+,-\}, F_{\alpha \beta}$ is the field strength, $\gamma_{(2)}^{\alpha}$ the two-dimensional representation of the gamma matrices, and $D_{\alpha}=\partial_{\alpha}+\mathrm{i} e A_{\alpha}$. In light-cone gauge $\left(A_{-}=0\right), \psi_{-}$is the left moving part of a free massless fermi field.

$$
\psi_{-}=\psi_{-}^{0}\left(x^{+}\right)
$$

That is true however the theory is quantized. If it is quantized on $x^{+}=0$, it is the only solution to the constraint equation

$$
\partial_{-} \psi_{-}=0
$$

The bosonized form is

$$
\psi_{-}^{0}=Z_{-} \mathrm{e}^{\Lambda_{-}^{(-)}} \sigma_{-} \mathrm{e}^{\Lambda_{-}^{(+)}},
$$


where $\Lambda_{-}$is a bosonic field depending on $x^{+}$(it is composed entirely of unphysical operators and physical states do not contain quanta from it), $\sigma_{-}$is a (space-time independent) spurion and $Z_{-}$is a wave function renormalization constant. $(+)$and (-) refer to positive and negative frequency parts. We write $\psi_{+}$similarly in bosonized form as

$$
\psi_{+}=Z_{+} \mathrm{e}^{\Lambda_{+}^{(-)}} \sigma_{+} \mathrm{e}^{\Lambda_{+}^{(+)}} .
$$

The physical vacuum in this theory is known exactly:

$$
|\Omega(\theta)\rangle \equiv \sum_{M=-\infty}^{\infty} \mathrm{e}^{\mathrm{i} M \theta}|\Omega(M)\rangle \quad ; \quad|\Omega(M)\rangle=\left(\sigma_{+}^{*} \sigma_{-}\right)^{M}|0\rangle
$$

where $\sigma^{-1} \equiv \sigma^{*}$. This same state is found whether the system is quantized on $x^{+}=0$ or on $x^{0}=0$. The existence of these vacua, and their form, is determined from the fact that we have residual gauge transformations in lightcone gauge and that $\sigma_{+}^{*} \sigma_{-}$is their generator. The vacuum has the chiral condensate

$$
\langle\Omega(\theta)|\bar{\psi} \psi| \Omega(\theta)\rangle=-\frac{e}{2 \pi^{3 / 2}} e^{\gamma} \cos \theta
$$

If the bare mass is nonzero there exists an induced interaction [19].

The case of the Schwinger model quantized on the light-cone with antiperiodic boundary conditions in $x^{-}$has also received a thorough discussion in the literature [3, 5]. The vacuum is exactly known also for Adjoint $Q C D_{1+1}$, where again it is determined by residual gauge invariance [6].

[1] S. J. Brodsky, H-C. Pauli, and S. Pinsky, Phys. Rep. 301 (1998) 299

[2] M. Shifman, in ITEP Lectures on Particle Physics and Field Theory (World Scientific, Singapore, 1999)

[3] G. McCartor, Z.Phys. C64 (1994) 349

[4] Y. Nakawaki and G. McCartor, Prog. Theor. Phys. 103, 161 (2000).

[5] G. McCartor, Nuc. Phys. B(Proc. Suppl)90 (2000) 37

[6] G. McCartor, D. Robertsons, S. Pinsky, Phys. Rev. D 561035 (1997)

[7] K. Wilson et al., Phys. Rev. D49 (1994) 6720;

B. H. Allen and R. J. Perry, Phys. Rev. D62 (2000) 025005 
[8] S. Dalley and B. van de Sande, Phys. Rev. Lett. 82 (1999) 1088; Phys. Rev. D62 (2000) 014507

[9] E. Prokhvatilov and V. Franke, Yad. Fiz. 49 (1989) 1109;

F. Lenz, M. Thies, S. Levit, and K. Yazaki, Ann. Phys. 208 (1991) 1;

E.-M. Ilgenfritz, Yu. P. Ivanov, and H-J. Pirner, Phys. Rev. D62 (2000) 054006

[10] F. Lenz, M. Thies, and K. Yazaki, Phys. Rev. D63 (2001) 045018;

M. Burkardt, F. Lenz, and M. Thies, Phys. Rev. D65 (2002) 125002;

F. Lenz and D. Steinbacher, Phys. Rev. D67 (2003) 045010

[11] Y. Kim, S. Tsujumaru, and K. Yamawaki, Phys. Rev. Lett. 74, 4771(1995);

M. Burkardt, Phys. Rev. D58 (1998) 096015.

[12] D. Mustaki, Bowling Green State University preprint, hep-ph/9404206.

[13] G. McCartor, Z. Phys. C41 (1988) 271.

[14] H. Leutwyler, Phys. Lett. B48 (1974) 431; Nucl. Phys. B76 (1974) 413.

[15] R. Perry, Nucl. Phys. B (Proc. Suppl.) 90 (2000) 87.

[16] J. H. Lowenstein and J. A. Swieca, Ann. Phys. 68 (1971) 172.

[17] Y. Nakawaki, Prog. Theor. Phys.72 (1984)134; for the discrete case see Prog. Theor. Phys. 70 1105, (1983).

[18] B. Klaiber, Boulder Lectures in Theoretical Physics (Gordon and Breach, 1967), vol. XA.

[19] G. McCartor, Phys. Rev. D60 (1999) 105004

[20] A. Casher, Phys. Rev. D 14 (1976) 452;

T. Maskawa and K. Yamawaki, Prog. Theor. Phys. 56 (1976) 270.

C. Thorn, Phys. Lett. B 70 (1977) 77;

H.-C. Pauli and S. J. Brodsky, Phys. Rev. D 32 (1985) 1993

[21] F. Antonuccio and S. Dalley, Nucl.Phys. B461 (1996) 275; Phys.Lett. B376 (1996) 154.

[22] J. Alfaro, A. Andrianov, and P. Labrana, JHEP 0407 (2004) 067.

[23] M. Burkardt, Phys. Rev. D57 (1998) 1136

[24] M. Burkardt and S. Dalley, Prog. Part. Nucl. Phys. 48317 (2002);

S. Dalley and B. van de Sande, in Lightcone Physics: Hadrons and Beyond p78 (Durham IPPP, 2003) hep-ph/0311368.

[25] M. Burkardt and H. El-Khozondar, Phys. Rev. D60, 054504 (1999);

S. Dalley, Phys. Rev. D64, 036006 (2001);

M. Burkardt and S.K.Seal, Phys. Rev. D65, 034501 (2002); 
S. Dalley and B. van de Sande, Phys. Rev. D67, 114507 (2003)

[26] S.A. Paston, V.A. Franke, and E.V. Prokhvatilov, Teor. Mat. Fiz. 120 (1999), 417 arXiv:hep-th/0002062.

[27] F. Lenz, K. Ohta, M. Thies, and K. Yazaki, Phys. Rev. D70 (2004) 025015

[28] S.A. Paston, V.A. Franke, and E.V. Prokhvatilov, Phys. Atom. Nucl. 68 (2005) 267 